\title{
Deformation Quantization in White Noise Analysis ${ }^{\star}$
}

\author{
Rémi LÉANDRE
}

Institut de Mathématiques, Université de Bourgogne, 21000 Dijon, France

E-mail:Remi.Leandre@u-bourgogne.fr

Received August 02, 2006, in final form January 30, 2007; Published online February 21, 2007

Original article is available at http://www.emis.de/journals/SIGMA/2007/027/

\begin{abstract}
We define and present an example of a deformation quantization product on a Hida space of test functions endowed with a Wick product.
\end{abstract}

Key words: Moyal product; white noise analysis

2000 Mathematics Subject Classification: 53D55; 60H40

\section{Introduction}

This work follows the work of Dito-Léandre [7] which was using tools of the Malliavin Calculus in order to define a Moyal product on a Wiener space.

Let us consider a finite-dimensional symplectic manifold $M$. It inherits from the symplectic form $\omega$ a Poisson structure $\{\cdot, \cdot\}$ whose matrix is the inverse of the matrix of the symplectic structure. The deformation quantization program of a Poisson manifold was initiated by BayenFlato-Fronsdal-Lichnerowicz-Sternheimer [1, 2, 8, 17, 23]. The simplest case to study is the case of $\mathbb{R}^{n} \oplus \mathbb{R}^{n *}$ endowed with its constant natural symplectic structure. This leads in particular to the notion of Moyal product.

In the case of a Hilbert space $H$, Dito [6] defines deformation quantization on a Hilbert space $H \oplus H^{*}$ endowed with its constant canonical symplectic structure and he defines a Moyal product on it on an appropriate algebra of functions. The main remark is that the constant matrix of the associated Poisson structure is still bounded.

This permits Dito-Léandre [7] to define the Moyal product on $W \oplus W^{*}$ where $W$ is an abstract Wiener space. The constant symplectic form is the standard one on $H \oplus H^{*}$, the underlying Hilbert space of $W \oplus W^{*}$, such that the constant matrix of Poisson structure is still bounded. In that work, they consider the algebra of functionals smooth in the Malliavin sense $[15,18,19,20,22]$ in order to define the Moyal product on $W \oplus W^{*}$.

We consider in this work the case where the underlying Hilbert space of the theory is a Sobolev Hilbert space of maps from the circle into $\mathbb{R}^{n}$ endowed with a constant symplectic structure. We do not consider the standard 2-form in order to do the quantization, but another form which is still interesting to consider. The constant matrix of the involved Poisson structure is unbounded so that we cannot use the construction of [7]. This leads to some modifications:

- We replace the algebra of functionals of Malliavin type by a Hida test functional space $[3,11,12,21]$.

- We replace the Wiener product by the normalized Wick product [11].

The behaviour of our theory is completely different of the behaviour in field theory of the classical Garding-Wightman result [10] for Canonical Commutation Relations in infinite dimension.

${ }^{\star}$ This paper is a contribution to the Proceedings of the Workshop on Geometric Aspects of Integrable Systems (July 17-19, 2006, University of Coimbra, Portugal). The full collection is available at http://www.emis.de/journals/SIGMA/Coimbra2006.html 
This is related to the fact that the Hida test algebra is a space of continuous functionals on an abstract Wiener space associated to the Hilbert space of the theory. The Hida test algebra is so small that quantities as white noise behaves as if we were in finite dimension. Nevertheless, there is a tentative to interpret some quantum fields by using tools of white noise analysis [13, 14].

White noise analysis was created in order to understand very singular objects as, for instance, the speed of the Brownian motion. This explain that we get equivalences of some deformation quantization in white noise analysis, which were inequivalent in the theory of [6].

Our motivation comes from field theory [4, 5, 9, 24]. We choose a simple model in this approach, but it should be possible to consider more complicated Gaussian models. We have chosen this simple model in order to get simple computations. On the other hand, the free loop space is a well-known object of conformal field theory and string theory.

\section{Deformation quantization in white noise analysis}

Let $H\left(S^{1} ; \mathbb{R}^{n}\right)$ be the Hilbert space of maps $\gamma$ from the circle into $\mathbb{R}^{n}$ endowed with its canonical Hilbert structure such that

$$
\int_{0}^{1}|\gamma(s)|^{2} d s+\int_{0}^{1}\left|\gamma^{\prime}(s)\right|^{2} d s=\|h\|^{2}<\infty .
$$

We get by Fourier expansion an orthonormal basis $\gamma_{k, i}$ of this Hilbert space:

$$
\gamma_{k, i}(s)=\left(\sqrt{C_{1} k^{2}+1}\right)^{-1} e_{i} \cos (2 \pi k s)
$$

if $k \geq 0$ and if $k<0$

$$
\gamma_{k, i}(s)=\left(\sqrt{C_{1} k^{2}+1}\right)^{-1} e_{i} \sin (2 \pi k s),
$$

where $e_{i}$ is the canonical basis of $\mathbb{R}^{n}$.

We consider a multiindex $I=\left(\left(k_{1}, i_{1}\right), \ldots,\left(k_{|I|}, i_{|I|}\right)\right.$. We introduce the Hida weight:

$$
w_{r}(I)=\prod\left(\sqrt{C_{1} k_{i}^{2}+1}\right)^{r}
$$

associated to this multiindex. $F^{I}$ denotes the normalized symmetric tensor product of the $\gamma_{k_{i}, e_{i}}$ associated to this multiindex.

We consider the weighted Fock space $W \cdot N_{r, C}$ of series

$$
\sum b_{I} F^{I}=F
$$

such that

$$
\|F\|_{r, C}^{2}=\sum\left|b_{I}\right|^{2} w_{r}(I) C^{|I|}<\infty
$$

where $b_{I} \in \mathbb{C}$. In order to avoid some redundancies, we order the multiindices by lexicographic order such that after this choice $F$ is written in a unique way.

Definition 1. The Hida test function $W \cdot N_{\infty-}$ space is the intersection of all $W \cdot N_{r, C}$ for $r>0$ and $C>0$ endowed with its natural topology. 
It is a Fréchet space. Since the system of norms $\|\cdot\|_{r, C}$ increase with $r$ and $C$, it is a projective limit of Hilbert spaces. Moreover if $r$ and $C$ are large, the imbedding of $W \cdot N_{r, C}$ in $W \cdot N_{r^{\prime}, C^{\prime}}$ is Hilbert-Schmidt for large $r^{\prime}$ and $C^{\prime}$. This follows from the classical fact that

$$
\sum w_{r}(I)^{-1} C^{|I|}<\infty
$$

if $r$ is large enough and $C$ small. This shows that $W \cdot N_{\infty-}$ is a nuclear space.

We define the Wick product : $F^{I_{1}} F^{I_{2}}$ : as the normalized symmetric tensor product of all the $\gamma_{k, i}$ in the concatenation of the multiindices $I_{1}$ and $I_{2}$.

Theorem 1. $W \cdot N_{\infty-}$ is a commutative algebra for the Wick product.

Proof. Let

$$
F_{1}=\sum b_{I}^{1} F^{I}, \quad F_{2}=\sum b_{I}^{2} F^{I} .
$$

Therefore

$$
F_{3}=: F_{1} F_{2}:=\sum b_{I}^{3} F^{I},
$$

where

$$
a_{I}^{3}=\sum_{I_{1}, I_{2}} b_{I_{1}}^{1} b_{I_{2}}^{2}
$$

where the sum runs over all considered multiindices $I_{1}$ and $I_{2}$ whose concatenation is $I$. If $I_{1}$ and $I_{2}$ are such multiindices, we have clearly

$$
w_{r}(I)=w_{r}\left(I_{1}\right) w_{r}\left(I_{2}\right) .
$$

Moreover there are at most $C^{|I|}=C^{\left|I_{1}\right|} C^{\left|I_{2}\right|}$ terms in the sum (1) such that

$$
\left|b_{I}^{3}\right|^{2} \leq C \sum_{I_{1}, I_{2}}\left(\left|b_{I_{1}}^{1}\right|^{2} C^{\left|I_{1}\right|}\right)\left(\left|b_{I_{2}}^{2}\right|^{2} C^{\left|I_{2}\right|}\right) .
$$

(We refer to [16] for an analogous statement.)

Definition 2. A Poisson structure on $W \cdot N_{\infty-}\{\cdot, \cdot\}$ is given by a $\mathbb{C}$-bilinear map $\{\cdot, \cdot\}$ from $W N_{\infty-} \times W N_{\infty-}$ into $W \cdot N_{\infty-}$ such that:

(i) $\{\cdot, \cdot\}$ is antisymmetric, satisfies the Jacobi derivation and is a derivation with respect of the Wick product in each argument;

(ii) if $F_{1}=1,\left\{F_{1}, F_{2}\right\}=0$;

(iii) for all $r$ and $C$, there exist $r_{1}$ and $C_{1}$ such that

$$
\left\|\left\{F_{1}, F_{2}\right\}\right\|_{r, C} \leq K\left\|F_{1}\right\|_{r_{1}, C_{1}}\left\|F_{2}\right\|_{r_{1}, C_{1}} .
$$

In this formalism we can easily consider the deformation quantization of $[1,2,8,17,23]$.

We consider the set of formal series $W \cdot N_{\infty-}[[h]]$ with coefficients in the Hida test functional space.

Definition 3. A star-product on $W \cdot N_{\infty-}[[h]]$ is a $\mathbb{C}[[h]]$-bilinear product $*_{h}$ on $W N_{\infty-}[[h]] \times$ $W \cdot N_{\infty-}[[h]]$ with values in $W \cdot N_{\infty-}[[h]]$ given by

$$
F_{1} *_{h} F_{2}=\sum_{l \geq 0} h^{l} P_{l}\left(F_{1}, F_{2}\right)
$$

for $F_{1}$ and $F_{2}$ belonging to the Hida test functional space. The star-product is extended by $\mathbb{C}[[h]]$-bilinearity to $W \cdot N_{\infty-}[[h]]$ and satisfies if $F_{1}, F_{2}, F_{3}$ belong to $W \cdot N_{\infty-}$ : 
(i) $P_{0}\left(F_{1}, F_{2}\right)=: F_{1} F_{2}:$;

(ii) $P_{1}\left(F_{1}, F_{2}\right)-P_{1}\left(F_{2}, F_{1}\right)=2\left\{F_{1}, F_{2}\right\}$;

(iii) for all $r, C, l$, there exist $r_{1}, C_{1}$ such that

$$
\left\|P_{l}\left(F_{1}, F_{2}\right)\right\|_{r, C} \leq K\left\|F_{1}\right\|_{r_{1}, C_{1}}\left\|F_{2}\right\|_{r_{1}, C_{1}}
$$

and $P_{l}$ vanishes on constants.

(iv) $F_{1} *_{h}\left(F_{2} *_{h} F_{3}\right)=\left(F_{1} *_{h} F_{2}\right) *_{h} F_{3}$.

\section{Example: the Hida star product}

Let $\omega=\omega_{i, j}$ be a nondegenerate antisymmetric bilinear form on $\mathbb{R}^{n}$ ( $n$ is even). Without loss of generality, we can write $\omega$ as:

$$
\omega_{2 i, 2 i+1}=-\omega_{2 i+1,2 i}=1
$$

and $\omega_{i, j}=0$ elsewhere. Namely, this diagonalization do not change the Hida space of test functionals we have considered in this paper. This comes from the fact that a linear transformation on $\mathbb{R}^{n}$ induces a linear transformation on $\left(\mathbb{R}^{n}\right)^{\hat{\otimes} r}$, the symmetric tensor product of $\mathbb{R}^{n}$ of length $r$ of norm bounded by $C^{k}$. If we perform a linear change of coordinates on $\mathbb{R}^{n}$, this induces on the system of $F_{I}|I|=r$ a linear transformation. But it is a linear transformation on each block realized by the $F^{I} I=\left(k_{I}, i_{I}\right), k_{I}$ being fixed. Each block is identified with $\left(\mathbb{R}^{n}\right)^{\hat{\otimes} r}$, where we can look at the induced linear transformation. But, we could repeat the following considerations without using this diagonalization of $\omega$.

We introduce the antisymmetric bilinear form on $H\left(S^{1} ; \mathbb{R}^{n}\right)$

$$
\Omega\left(\gamma^{1}, \gamma^{2}\right)=\int_{0}^{1} \omega\left(\gamma^{1}(s), \gamma^{2}(s)\right) d s, \quad \Omega=\Omega_{\left(k_{1}, i_{1}\right),\left(k_{2}, i_{2}\right)},
$$

where

$$
\Omega_{(k, 2 i),(k, 2 i+1)}=\left(C k^{2}+1\right)^{-1}=-\Omega_{(k, 2 i+1),(k, 2 i)}
$$

and other components of $\Omega$ elsewhere vanish. This antisymmetric bilinear form leads to a singular Poisson structure whose matrix form is $\Omega^{-1}$ which is not bounded: therefore the theory of [7] is not suitable to describe the deformation quantization theory related to this symplectic structure.

Let $a_{(k, i)}$ be the annihilation operator on the symmetric Fock space associated to $\gamma_{(k, i)}$. We have that:

$$
a_{\left(k_{1}, i_{1}\right)} \cdots a_{\left(k_{l}, i_{l}\right)} F^{I}=C\left(I_{1},\left(k_{1}, i_{1}\right), \ldots,\left(k_{l}, i_{l}\right)\right) F^{I_{1}},
$$

where in $I_{1}$ we have removed $\left(k_{1}, i_{1}\right), \ldots,\left(k_{l}, i_{l}\right)$ if it is possible (in the other case the expression vanishes). We have the bound

$$
\left|C\left(I_{1},\left(k_{1}, i_{1}\right), \ldots,\left(k_{l}, i_{l}\right)\right)\right| \leq C_{l}^{|I|} .
$$

The constant $C\left(I_{1},\left(k_{1}, i_{1}\right), \ldots,\left(k_{l}, i_{l}\right)\right)$ comes from the fact that we are considering the Bosonic Fock space: if we consider the same Boson at the power $n$, the associated annihilation operator transforms it in $n$-times the Boson at the power $n-1$.

We consider finite sums $F_{1}=\sum b_{I}^{1} F^{I}$ and $F_{2}=\sum b_{I}^{2} F^{I}$. In a traditional way, we can put

$$
\left\{F_{1}, F_{2}\right\}=\sum \Omega^{\left(k_{1}, i_{1}\right),\left(k_{2}, i_{2}\right)}: a_{\left(k_{1}, i_{1}\right)} F_{1} a_{\left(k_{2}, i_{2}\right)} F_{2}: .
$$


(We consider normalized Wick products.) $\Omega^{\left(k_{1}, i_{1}\right),\left(k_{2}, i_{2}\right)}$ are the generic elements of the inverse of the symplectic form $\Omega$ and are not bounded. Therefore $\{\cdot, \cdot\}$ do not act on the Sobolev spaces of the Malliavin Calculus unlike the Poisson structure studied in [7], and we have to consider different functional spaces if we want to extend the previous formula from finite sums to series. We have:

Proposition 1. $\{\cdot, \cdot\}$ defines a Poisson structure in the sense of Definition 3 on $W \cdot N_{\infty-}$.

Proof. Let us show first (iii) in Definition 3. We have

$$
\left\{F_{1}, F_{2}\right\}=\sum b_{I}^{3} F^{I}
$$

where

$$
b_{I}^{3}=\sum\left(C k^{2}+1\right) b_{I_{1} \cup(k, 2 i)}^{1} b_{I_{2} \cup(k, 2 i+1)}^{2} C\left(I_{1},(k, 2 i)\right) C\left(I_{2},(k, 2 i+1)\right)+A,
$$

where $A$ is a similar term and where we sum over all $k, i, I_{1}, I_{2}$ so that the concatenation $I_{1} \cup I_{2}$ of $I_{1}, I_{2}$ is equal to $I$. We apply Cauchy-Schwartz inequality in $(k, i)$, we use the bound of $C\left(I_{1},(k, 2 i)\right), C\left(I_{2},(k, 2 i+1)\right)$ given in (3) to get

$$
\begin{aligned}
\omega_{r}(I)\left|b_{I}^{3}\right|^{2} \leq & K \sum_{k, k^{\prime}, i, i^{\prime}}\left(\omega_{r_{1}}\left(I_{1} \cup(k, 2 i)\right)\left|b_{I_{1} \cup(k, 2 i)}^{1}\right|^{2} C_{1}^{\left|I_{1}\right|+1}\right) \\
& \times\left(\omega_{r_{1}}\left(I_{2} \cup\left(k^{\prime}, 2 i^{\prime}+1\right)\right)\left|b_{I_{2} \cup\left(k^{\prime}, 2 i^{\prime}+1\right)}^{2}\right|^{2} C_{1}^{\left|I_{2}\right|+1}\right)
\end{aligned}
$$

for $r_{1}$ and $C_{1}$ large enough, where we sum on the set of multiindices $I_{1}$ and $I_{2}$ is such that $I_{1} \cup I_{2}=I$. We do the same for $A$ in (4). We use for that

$$
\sum\left(C k^{2}+1\right)^{-r^{\prime}}<\infty
$$

if $r^{\prime}>1$. We deduce from the previous inequality that

$$
\left\|\left\{F_{1}, F_{2}\right\}\right\|_{r, C} \leq K\left(\sum \omega_{r_{1}}(I)|I| C_{1}^{|I|}\left|b_{I}^{2}\right|^{2}\right)\left(\sum \omega_{r_{1}}(I)|I| C_{1}^{|I|}\left|b_{I}^{2}\right|^{2}\right) .
$$

Therefore we deduce that

$$
\left\|\left\{F_{1}, F_{2}\right\}\right\|_{r, C} \leq K\left\|F_{1}\right\|_{r_{1}, C_{1}^{\prime}}\left\|F_{2}\right\|_{r_{1}, C_{1}^{\prime}}
$$

for $r_{1}, C_{1}^{\prime}$ large enough. This shows (iii).

The algebraic properties of the Poisson product arise from the fact that the family of annihilation operators commute and an annihilation operator is a derivation for the Wick product.

If $F_{1}$ and $F_{2}$ are finite sums, we can define as usual by using the Wick product:

$$
\begin{aligned}
F_{1} *_{h} F_{2}= & \sum_{l \geq 0}(-h / 2)^{l} l !^{-1} \sum \Omega^{\left(k_{1}, i_{1}\right),\left(k_{1}^{\prime}, i_{1}^{\prime}\right)} \cdots \Omega^{\left(k_{l}, i_{l}\right),\left(k_{l}^{\prime}, i_{l}^{\prime}\right)} \\
& \times: a_{\left(k_{1}, i_{1}\right)} \cdots a_{\left(k_{l}, i_{l}\right)} F_{1} a_{\left(k_{1}^{\prime}, i_{1}^{\prime}\right)} \cdots a_{\left(k_{l}^{\prime}, i_{l}^{\prime}\right)} F_{2}: .
\end{aligned}
$$

The sum is in fact finite since $F_{1}$ and $F_{2}$ are finite sums. It is the exponential of the Poisson Bracket. Let us stress the difference with [7]: in [7], we were considering the canonical symplectic form on $W \oplus W^{*}$ whose inverse is bounded, and so the Moyal product of [7] was acting on the big space of Malliavin test functionals. Here it is not the case. Let us recall the main difference between the Malliavin test algebra and the Hida test algebra. The Hilbert space $H\left(S^{1} ; \mathbb{R}^{n}\right)$ induces a Gaussian measure on the Banach space $B$ of continuous functions from $S^{1}$ 
into $\mathbb{R}^{n}$. The ordinary Fock space $W \cdot N_{0,1}$ coincides with the $L^{2}$ of this Gaussian measure. The Malliavin test algebra is constituted of functionals almost surely defined on $B$ and the Hida test algebra is constituted of continuous functionals on $B$. In order to stress the difference, let us

consider chaos of length $1, F=\sum_{|I|=1} b_{I} F^{I}$. These chaoses of length 1 belong to the Malliavin algebra if and only if $\sum\left|b_{I}\right|^{2}<\infty$ because the $L^{p}$ norms and the $L^{2}$ norms are equivalent on an abstract Wiener space for Wiener chaos of bounded norm. It is therefore clear that our Poisson structure does not act on the restriction of the Malliavin algebra constituted of chaoses of length 1.

Theorem 2. Formula (5) can be extended in a star-product in the sense of Definition 3. We call it the Hida star product associated to the symplectic structure given by $\Omega$.

Proof. The algebra is the same as in the classical case [6]. Only the analysis is different. We put

$$
P_{l}\left(F_{1}, F_{2}\right)=\sum b_{I}^{3} F^{I}
$$

where $b_{I}^{3}$ is a sum of a bounded terms of the following type:

$$
\begin{aligned}
A= & \sum\left(C k_{1}^{2}+1\right) \cdots\left(C k_{l}^{2}+1\right) b_{I_{1} \cup\left(k_{1}, 2 i_{1}\right) \cup \cdots \cup\left(k_{l}, 2 i_{l}\right)}^{1} \\
& \times C\left(I_{1},\left(k_{1}, 2 i_{1}\right), \ldots,\left(k_{l}, 2 i_{l}\right)\right) b_{I_{2} \cup\left(k_{1}^{\prime}, 2 i_{1}^{\prime}+1\right) \cup \cdots \cup\left(k_{l}^{\prime}, 2 i_{l}^{\prime}+1\right)}^{2} \\
& \times C\left(I_{2},\left(k_{1}^{\prime}, 2 i_{1}^{\prime}+1\right), \ldots,\left(k_{l}^{\prime}, 2 i_{l}^{\prime}+1\right)\right),
\end{aligned}
$$

where we sum on all $k_{i}, i_{l}, i_{l}^{\prime}$ and all multiindices $I_{1}$ and $I_{2}$ such that their concatenation $I_{1} \cup I_{2}$ equals $I$. By doing as before and using the estimates (3), we deduce that

$$
\begin{aligned}
\omega_{r}(I)|A|^{2} \leq & K \sum\left(\omega_{r_{1}}\left(I_{1} \cup\left(k_{1}, 2 i_{1}\right) \cup \cdots \cup\left(k_{l}, 2 i_{l}\right)\right) C_{1}^{\left|I_{1}\right|+l}\left|b_{I_{1} \cup\left(k_{1}, 2 i_{1}\right) \cup \cdots \cup\left(k_{l}, 2 i_{l}\right)}^{1}\right|^{2}\right) \\
& \times\left(\omega_{r_{1}}\left(I_{2} \cup\left(k_{1}^{\prime}, 2 i_{1}^{\prime}+1\right) \cup \cdots \cup\left(k_{l}^{\prime}, 2 i_{l}^{\prime}+1\right)\right) C_{1}^{\left|I_{2}\right|+l}\left|b_{I_{2} \cup\left(k_{1}^{\prime}, 2 i_{1}^{\prime}\right) \cup \cdots \cup\left(k_{l}^{\prime}, 2 i_{l}^{\prime}+1\right)}^{2}\right|^{2}\right),
\end{aligned}
$$

where we sum on all $\left(k_{l}, 2 i_{l}\right)$, all $\left(k_{l^{\prime}}, 2 i_{l^{\prime}}+1\right)$ and all multiindices $I_{1}$ and $I_{2}$ such that $I_{1} \cup I_{2}=I$.

We deduce that:

$$
\left\|P_{l}\left(F_{1}, F_{2}\right)\right\|_{r, C}^{2} \leq K\left(\sum \omega_{r_{1}}(I) C_{1}^{|I|}|I|^{l}\left|b_{I}^{1}\right|^{2}\right)\left(\sum \omega_{r_{1}}(I) C_{1}^{|I|}|I|^{l}\left|b_{I}^{2}\right|^{2}\right)
$$

for $r_{1}$ and $C_{1}$ large enough such that

$$
\left\|P_{l}\left(F_{1}, F_{2}\right)\right\|_{r, C} \leq K\left\|F_{1}\right\|_{r_{1}, C_{1}^{\prime}}\left\|F_{2}\right\|_{r_{1}, C_{1}^{\prime}}
$$

from which the result follows.

\section{Equivalence of deformations}

The main difference between this work and $[6,7]$ is that the space of Hida test functionals is very small, hence the space of allowed deformation is very big. This implies that some inequivalent deformations in the theory of [6] are here equivalent. In order to stress the difference, we will take the model of [7].

$H$ is the Hilbert space of maps from $[0,1]$ into $\mathbb{R}$ such that

$$
\|\gamma\|_{0}^{2}=\int_{0}^{1}|d / d s \gamma(s)|^{2} d s<\infty .
$$


We consider $H \oplus H^{*}=H_{t}$ endowed with its canonical symplectic form. We define $W_{\infty-}$ to be the space of maps such that $\int_{0}^{1}\left|d^{r} / d s^{r} \gamma(s)\right|^{2} d s<\infty$ for all $r$. It is a Fréchet space. We choose a convenient Hilbert basis of $H$ : if $n>0, \gamma_{n}(s)=\frac{\sin [2 \pi n s]}{C_{1} n}$ and if $n<0, \gamma_{n}(s)=\frac{\cos [2 \pi n s]-1}{C_{1} n}$. Associated to this Hilbert space, and by using the convenient Hida weights associated to this basis, we can define the Hida test algebra $W \cdot N_{\infty-}$. We can define a Poisson structure $\{\cdot, \cdot\}$ associated to this symplectic form on $H_{t}$ which acts continuously on $W \cdot N_{\infty-} \times W \cdot N_{\infty-}$. Computations are similar to the Part III, but simpler since the matrix of the Poisson structure is bounded.

Let $\gamma_{1} \oplus \gamma_{2}$ belong to $W_{\infty_{-}} \oplus W_{\infty_{-}}$. We consider the Wick exponential $\Phi_{\gamma_{1}, \gamma_{2}}$

$$
h_{1} \oplus h_{2} \rightarrow: \exp \left[\left\langle h_{1}, \gamma_{1}\right\rangle_{0}+\left\langle h_{2}, \gamma_{2}\right\rangle_{0}\right]: \text {. }
$$

The Wick exponentials are dense in $W \cdot N_{\infty-}$.

We consider an operator $A: \gamma_{n} \rightarrow \lambda_{n} \gamma_{n}$ with $\left|\lambda_{n}\right| \leq C|n|^{\alpha}$. According to [6], we put

$$
E_{A}\left[F_{1}, F_{2}\right]=\sum: a_{i}^{1} F_{1} \lambda_{i} a_{i}^{2} F_{2}:+\sum: a_{i}^{1} F_{2} \lambda_{i} a_{i}^{2} F_{1}:
$$

where $a_{i}^{1}$ are the standard annihilation operators in the direction of $H$ associated to $\gamma_{i}$ and $a_{i}^{2}$ are standard annihilation operators in the direction of $H^{*} \sim H$. Since $\left|\lambda_{i}\right|$ are bounded by $C|i|^{\alpha}$ and we are considering the same Hida weight as in the first part, but with this new orthogonal basis, it follows that:

Theorem 3. $E_{A}$ is continuous from $W \cdot N_{\infty-} \times W \cdot N_{\infty-}$ into $W \cdot N_{\infty_{-}}$.

We put according to [6],

$$
C_{1}^{A}\left[F_{1}, F_{2}\right]=\left\{F_{1}, F_{2}\right\}+E_{A}\left[F_{1}, F_{2}\right]
$$

and $C_{r}^{A}\left[F_{1}, F_{2}\right]=\left(C_{1}^{A}\right)^{r}\left[F_{1}, F_{2}\right]$ in the sense of bidifferential operators. $C_{r}^{A}$ is still continuous from $W \cdot N_{\infty_{-}} \times W \cdot N_{\infty_{-}}$into $W \cdot N_{\infty_{-}}$(the proof is very similar to the proof of Theorem 1 ).

Definition 4. We put

$$
F_{1} *_{h}^{A} F_{2}=: F G:+\sum_{r \geq 1} \frac{h^{r}}{r !} C_{r}^{A}\left(F_{1}, F_{2}\right) .
$$

As in Theorem 2, due to the polynomial growth of the $\lambda_{i}, *_{h}^{A}$ defines a quantization by deformation in Hida sense of $\{\cdot, \cdot\}$. But unlike in [6], we have:

Proposition 2. $*_{h}^{A}$ and $*_{h}$ are equivalent on the Hida test functional space.

Proof. We put as in [6]

$$
T_{1} F=-\sum \lambda_{i} a_{i}^{1} a_{i}^{2} F
$$

Due to the polynomial growth of the $\lambda_{i}, T^{\prime}=\exp \left[h T_{1}\right]$ is continuous on $W \cdot N_{\infty-}[[h]]$ Moreover, let us recall that by [6] formula (2)

$$
\left.\left.\Phi_{\gamma_{1}, \gamma_{2}} *_{h}^{A} \Phi_{\gamma_{1}^{\prime}, \gamma_{2}^{\prime}}=\exp \left[h\left(\left\langle\gamma_{2}^{\prime},(A+\mathbb{I}) \gamma_{1}\right\rangle_{0}+\right\rangle \gamma_{2},(A+\mathbb{I}) \gamma_{1}^{\prime}\right\rangle_{0}\right)\right] \Phi_{\gamma_{1}+\gamma_{1}^{\prime}, \gamma_{2}+\gamma_{2}^{\prime}} .
$$

We conclude as in [6], by remarking that

$$
T^{\prime}\left(\Phi_{\gamma_{1}, \gamma_{2}} *_{h}^{A} \Phi_{\gamma_{1}^{\prime}, \gamma_{2}^{\prime}}\right)=T^{\prime} \Phi_{\gamma_{1}, \gamma_{2}} *_{h} T^{\prime} \Phi_{\gamma_{1}^{\prime}, \gamma_{2}^{\prime}}
$$

This proves the theorem since the Wick exponentials are dense in the Hida space. 
Remark 1. If $A=\mathbb{I}$, we get the normal product. Let us stress the difference with the theory of [6]. In [6], $*_{h}^{A}$ and $*_{h}$ were equivalent if and only if $A$ is a Hilbert-Schmidt operator, then the Moyal product and the normal product $(A=\mathbb{I})$ were inequivalent. In the case of $\mathbb{R}^{n}$, the Moyal product and the normal product are equivalent. For the Hida Calculus, deformation theory behaves more or less as in finite dimension. This comes from that the Hida test functional space is so small that all algebraic considerations in finite dimension, where we were considering finite sums, remain true in this context.

\section{References}

[1] Bayen F., Flato M., Fronsdal C., Lichnerowicz A., Sternheimer D., Deformation theory and quantization. I. Deformations of symplectic structures, Ann. Physics 111 (1978), 61-110.

[2] Bayen F., Flato M., Fronsdal C., Lichnerowicz A., Sternheimer D., Deformation theory and quantization. II. Physical applications, Ann. Physics 111 (1978), 111-151.

[3] Berezanskii Yu., Kondratiev Yu., Spectral methods in infinite-dimensional analysis, Vols. 1, 2, Kluwer, Dordrecht, 1995.

[4] Dito J., Star-product approach to quantum field theory: the free scalar field, Lett. Math. Phys. 20 (1990), 125-134.

[5] Dito J., Star-products and nonstandard quantization for Klein-Gordon equation, J. Math. Phys. 33 (1992), 791-801.

[6] Dito J., Deformation quantization on a Hilbert space, in Noncommutative Geometry and Physics, Editors Y. Maeda et al., World Scientific, Singapore, 2005, 139-157, math.QA/0406583.

[7] Dito G., Léandre R., A stochastic Moyal product on the Wiener space, J. Math. Phys., to appear.

[8] Dito J., Sternheimer D., Deformation quantization: genesis, developments and metamorphoses, in Deformation Quantization, Editor G. Halbout, IRMA Lectures Maths. Theor. Phys., Walter de Gruyter, Berlin, 2002, 9-54, math.QA/0201168.

[9] Duetsch M., Fredenhagen K., Perturbative algebraic field theory and deformation quantization, Fields Inst. Commun. 30 (2001), 151-160, hep-th/0101079.

[10] Garding L., Wightman A., Representations of the commutation relations, Proc. Natl. Acad. Sci. USA 40 (1954), 622-626.

[11] Hida T., Analysis of Brownian functionals, Carleton. Maths. Lect. Notes, Vol. 13, Ottawa, 1975.

[12] Hida T., Kuo H.H., Potthoff J., Streit L., White noise: an infinite dimensional calculus, Kluwer, Dordrecht, 1993.

[13] Huang Z., Luo S., Quantum white noises and free fields, Infin. Dimens. Anal. Quantum Probab. Relat. Top. 1 (1998) 68-82.

[14] Huang Z., Rang G., White noise approach to $\Phi_{4}^{4}$ quantum fields, Acta. Appl. Math. 77 (2003), $299-318$.

[15] Ikeda N., Watanabe S., Stochastic differential equations and diffusion processes, 2nd ed., North-Holland, Amsterdam, 1989.

[16] Léandre R., Rogers A., Equivariant cohomology, Fock space and loop groups, J. Phys. A: Math. Gen. 39 (2006), 11929-11946.

[17] Maeda Y., Deformation quantization and non commutative differential geometry, Sugaku Expositions 16 (1991), 224-255.

[18] Malliavin P., Stochastic calculus of variations and hypoelliptic operators, in Stochastic Analysis, Editor K. Itô, Kinokuyina, Tokyo, 1978, 155-263.

[19] Malliavin P., Stochastic analysis, Springer, Berlin, 1997.

[20] Nualart D., Malliavin calculus and related topics, Springer, Berlin, 1995.

[21] Obata N., White noise analysis and Fock space, Lect. Notes. Math., Vol. 1577, Springer, Berlin, 1994.

[22] Ustunel A.S., An introduction to analysis on Wiener space, Lect. Notes. Math., Vol. 1610, Springer, Berlin, 1995.

[23] Weinstein A., Deformation quantization. Séminaire Bourbaki, Astérisque 227. S.M.F., Paris (1994) 389-409.

[24] Witten E., Noncommutative geometry and string field theory, Nuclear Phys. B. 268 (1986), 253-294. 